\title{
Amigos con beneficios vs. sexo casual: definiendo sus comportamientos sexuales, amor, celos y creencias románticas*
}

\section{Friends with Benefits vs. Casual Sex: Defining their Sexual Behaviors, Love, Jealousy and Romantic Beliefs}

\author{
ENCARNACIÓN SORIANO-Ayala ${ }^{a}$ \\ Universidad de Almería, España \\ ORCID: http://orcid.org/0000-0002-9506-0625 \\ Herenía García-Serrán \\ Universidad de Almería, España \\ ORCID: http://orcid.org/0000-0002-9994-7389
}

a Autor de correspondencia. Correos electrónico: esoriano@ual.es

Para citar este artículo: Soriano-Ayala, E., \& GarcíaSerrán, H. (2019). Amigos con beneficios vs. sexo casual: definiendo sus comportamientos sexuales, amor, celos y creencias románticas. Universitas Psychologica, 18(2), 1-13. https://doi.org/10.11144/ Javeriana.upsy18-2.absc

\section{RESUMEN}

Esta investigación da a conocer los aspectos y características de las relaciones de amigos con beneficios comparándola con la práctica de sexo casual. Un total de 172 personas cumplimentaron un cuestionario en línea, en el que se solicitaba información acerca de las conductas sexuales, los tipos de amor y las creencias sobre el amor romántico. Los hallazgos revelan que las conductas sexuales y emocionales son más frecuentes en la relación de amigos con beneficios que en el sexo de una noche. Muestran un tipo de amor basado en la pasión y la intimidad, pero sin compromiso; sin embargo, mantienen creencias sobre el amor romántico. Estos resultados refuerzan el carácter híbrido de amigos con beneficios, a medio camino entre el vínculo puramente sexual y la relación romántica. Palabras clave

amigos con beneficios; sexo casual; conductas sexuales; amor; creencias románticas.

\section{ABSTRACT}

This research reveals the aspects and characteristics of the relationships of friends with benefits, comparing it with the practice of sex with unknown people. A total of 172 people completed an online questionnaire asking for information about their sexual behaviors, their love styles, and their beliefs about romantic love. The findings reveal that sexual and emotional practices are more prevalent in the relationship of friends with benefits than in one-night sex. Likewise, these subjects manifest a style of love based on passion and intimacy, but lacking commitment. Nevertheless, they keep romantic love beliefs. These results reinforce the hybrid character of friends with benefits, halfway between the purely sexual bond and the romantic relationship.

Keywords

friends with benefits; casual sex; sexual behaviors; love; romantic beliefs.

La sociedad actual cambia y avanza a un ritmo compulsivo e inconsistente. Vivimos en un mundo cada vez más 
individual y provisional en el que todos los ámbitos de la vida están envueltos en la inestabilidad y en la fugacidad (Bauman, 2001; Blandón-Hincapié \& López-Serna, 2016; Marquard, 2001). En esta sociedad, todo se vuelve líquido y evanescente, incluido el amor (Bauman, 2005). Están apareciendo nuevos conceptos de amor y de relaciones. En el panorama sexual destacan los vínculos fugaces, ajenos al amor y al romanticismo, denominados relaciones sexuales casuales u ocasionales (Dube, Lavoie, Blais, \& Hebert, 2017; Rodrigue \& Fernet, 2016; Wentland \& Reissing, 2014). Estas interacciones facilitan el acceso sexual sin formalismos, el amor deja de ser un componente básico de las relaciones sexuales y el comportamiento sexual no necesita del compromiso para producirse (Bauman, 2005; Furman \& Shaffer, 2011; Scott, Carrillo, \& Rivera, 2014).

Los estudios que se han realizado en España sobre las relaciones sexuales casuales abordan este tipo de relaciones de forma global, antagónica a la relación romántica y desde una perspectiva de salud sexual (Macchi, Benítez Leite, Núñez, \& Ortigoza, 2008; Rodríguez, Hernán, García, \& Romo, 2007; Uribe, Amador, Zacarías, \& Villarreal, 2012). Pocos trabajos se sumergen en lo variopinto del sexo casual, y cuando abordan específicamente las interacciones sexuales entre amigos, lo suelen hacer desde una perspectiva de Salud Pública (de Olalla, 2007; Fernández-Davila, 2007; Fuente, 2010; García-Serrán \& Soriano-Ayala, 2016).

En América Latina, diferentes trabajos enfatizan la pluralidad sexual entre los jóvenes, así como la dificultad para diferenciar estas relaciones y poner límites entre ellas (BlandónHincapié \& López-Serna, 2016; Gomes et al., 2017). Destacan los amigos con beneficios (AMB), amigos con derecho o "amigovios", relaciones abiertas que se caracterizan por la amistad y la ausencia de compromiso, las relaciones de "chocolate" referidas al primer vínculo afectivo, las "relaciones por calentura", breves y pasionales, y el "free" o encuentro erótico sexual (Hernández González \& Weiss, 2010; Vizzuetth, García, \& Guzmán, 2010).
En el ámbito anglosajón, se ha realizado un mayor número de estudios, que evidencia que el sexo desprovisto de amor es una relación común entre los adultos jóvenes, profundizando, además, en las diferentes variedades de relaciones sexuales ocasionales que se pueden llevar a cabo (Bisson \& Levine, 2009; Eisenberg, Ackard, Resnick, \& Neumark-Sztainer, 2009; Jonason, Li, \& Richardson, 2011; Manning, Giordano, \& Longmore, 2006; Wentland \& Reissing, 2014).

Se consideran las relaciones sexuales ocasionales como una categoría general integrada por una variedad de conexiones sexuales que transitan, como un continuo, desde un encuentro de una noche con un desconocido hasta una relación sexual con un amigo. Las dimensiones que diferencian las distintas experiencias sexuales son, el tipo de contacto (sexual, afectivo y social), la reiteración de los encuentros, la duración de la relación, el grado de intimidad y el tipo de socio (amigo, conocido y/o desconocido) (Claxton \& van Dulmen, 2013; Manning et al., 2006; Wentland \& Reissing, 2011).

\section{Amigos con beneficios y las conexiones sexuales de una noche}

$\mathrm{AMB}$ es un tipo de relación sexual ocasional en la que hay ausencia de fidelidad y de compromiso (Bisson \& Levine, 2009; Furman \& Shaffer, 2011; Lehmiller, VanderDrift, \& Kelly, 2011; Letcher \& Carmona, 2015; Lyons, Manning, Giordano, \& Longmore, 2010; Santos Vega, 2009; Scott, Hash, Stevens, \& Tejada, 2016); además, presenta particularidades, como el componente de la amistad, los rasgos de intimidad emocional y la pasión que la aproximan a una relación romántica (Owen, Fincham, \& Polser, 2017). Aunque el placer y la gratificación sexual es la meta de esta relación, los participantes obtienen además el soporte afectivo y social asociado a la amistad (García-Serrán \& Soriano-Ayala, 2016; Gusarova, Fraser, \& Alderson, 2012), aunque también, puede estar motivada por deseos románticos (Akbulut \& Weger, 2016; Bisson \& Levine, 2009). Desde una perspectiva dimensional, $\mathrm{AMB}$ se sitúa en 
uno de los extremos de las relaciones informales, con una dinámica más estable y próxima a una relación comprometida, mientras que el polo más inestable y efímero lo ocupa el contacto sexual con personas desconocidas, llamado aventura de una noche o "rollo" de una noche.

Las relaciones sexuales casuales incluyen una amplia gama de comportamientos sexuales, que van desde los besos hasta el coito (García \& Reiber, 2008; Hatfield, Hutchison, Bensman, Young, \& Rapson, 2012; Owen \& Fincham, 2011a, 2011b). Sin embargo, en AMB son más frecuentes los comportamientos afectivos (Grello, Welsh, \& Harper, 2006; Jonason, Li, $\&$ Cason, 2009; Jonason et al., 2011), ya que la amistad propicia una intimidad emocional de la que carecen otras relaciones ocasionales centradas estrictamente en la actividad sexual (Wentland \& Reissing, 2011, 2014).

Los trabajos de Sternberg (1997) señalan que diferentes tipos de relaciones deben mostrar diversos tipos de amor según los grados de intimidad, pasión y compromiso que se den en ellas. Dado que AMB posee características de una amistad y de una relación casual (Bisson \& Levine, 2009; Quirk, Owen, \& Fincham 2014), debe mostrar un importante componente pasional y de excitación sexual, un compromiso inexistente y una intimidad elevada, relacionada con la amistad (Lehmiller et al., 2011; McGinty, Knox, \& Zusman, 2007; Owen \& Fincham, 2011a; Quiñones, Martínez-Taboas, RodríguezGómez, \& Pando, 2017). En la relación sexual de una noche con personas anónimas o con personas que apenas se conocen, el motor principal, como se ha descrito en algunos estudios (Hatfield et al., 2012; Rodrigue \& Fernet, 2016; Smith et al., 2012), debe ser el despertar psicofisiológico y la pasión, mientras que la intimidad y el compromiso son inexistentes.

Tradicionalmente, la socialización de hombres y mujeres se ha realizado en torno al modelo de amor romántico (Suárez, 2016). Este modelo se construye sobre unas creencias aceptadas culturalmente, siendo su característica principal el amor de pareja ideal (Pascual, 2016; Suárez, 2016). Se cree que si se siente amor verdadero este será para toda la vida, capaz de superar obstáculos, de reafirmar la fidelidad y tendrá carácter omnipotente, que puede con todo (Ferrer, Bosch, \& Navarro, 2010), por lo que estas creencias tenderán a no estar presentes en las relaciones de AMB ni en el sexo casual, dadas las características que las definen. Los celos y la exclusividad, como respuesta que previene la infidelidad y la posible pérdida de la pareja (Yela, 2003; Pascual, 2016), no deben aparecer en el sexo casual ya que no existe exclusividad, respeto y fidelidad, propios de las relaciones comprometidas (Luiz de Andrade \& Wachelke, 2011).

La escasa evidencia empírica sobre las relaciones de AMB (Lehmiller, VanderDrift, \& Kelly, 2014) hace que este estudio se plantee la siguiente pregunta sobre las relaciones de AMB: ison relaciones estrictamente de sexo casual o llevan implícitos elementos que tradicionalmente han estado ligados a las relaciones románticas? El objetivo de este artículo es analizar los comportamientos sexuales, los tipos de amor y las creencias sobre el amor romántico que manifiestan los participantes implicados en una relación de $\mathrm{AMB}$ y los que mantienen sexo casual con personas extrañas o poco conocidas.

Las hipótesis de partida son:

1. En las relaciones de $A M B$ y en el sexo ocasional con extraños o poco conocidos, se exhibirán con la misma intensidad los comportamientos sexuales de tipo físico, pero sólo en las relaciones de AMB se expresarán con más intensidad conductas emocionales y de cercanía.

2. En las relaciones de AMB y en el sexo ocasional estará presente un tipo de amor apasionado y sin compromiso, y solo en AMB aparecerá el componente de la intimidad con más intensidad.

3. Las personas involucradas en una relación de $\mathrm{AMB}$ mostrarán más creencias sobre el amor romántico que los involucrados en relaciones ocasionales, siendo las mujeres las que mantengan estas creencias más que los hombres. 


\section{Método}

\section{Participantes}

La muestra estuvo compuesta por 172 participantes, de los cuales 119 (67.44\%) estaban implicados en AMB y 53 (30 815) en una relación sexual de una noche con un extraño o persona poco conocida. El $49.2 \%$ eran hombres y el $50.8 \%$ mujeres, y la media de edad fue de 23.3 años. La mayoría de los participantes, el 85 $\%$, eran heterosexuales, el $4.3 \%$ homosexuales, el $9.9 \%$ bisexuales y $0.8 \%$, pansexuales. En relación con el estado civil, el $96.95 \%$ eran solteros y el $3.05 \%$, divorciados. Atendiendo a la formación académica, el 49.95 \% habían cursado Enseñanza Secundaria Obligatoria y Bachiller, el $32.45 \%$ tenían estudios universitarios y el $17.6 \%$, formación profesional. La muestra fue seleccionada de manera autoelegida, ya que fue decisión de las personas invitadas participar o no en el estudio (Sterba \& Foster, 2008).

\section{Instrumentos}

Para llevar a cabo la investigación, se construyó un instrumento que aborda los datos personales y demográficos, tipo de relación, una escala con enunciados sobre comportamientos sexuales, la Escala Triangular del Amor de Sternberg (1986) y enunciados sobre los celos y creencias del amor romántico.

\section{Datos personales y sociodemográficos}

Se recogió información sobre la edad, el sexo, la nacionalidad, el estado civil, el nivel de estudios y la orientación sexual.

\section{Tipo de relación}

Se formuló la siguiente pregunta: ¿Qué tipo de relación sexual es la que tiene actualmente? a) Relación con un amigo o varios $(\mathrm{AMB})$ y

b) Relación casual de una noche con personas desconocidas o poco conocidas. Los participantes recibieron una definición de AMB extraída del estudio de Bisson y Levine (2009), 'cuando las personas que son amigos mantienen relaciones sexuales'.

\section{Comportamientos sexuales}

Escala elaborada por las autoras del artículo y formada por ítems inspirados en indicadores de los estudios de Bisson y Levine (2009) y Lehmiller et al. (2011). Con un grupo de nueve expertos en metodología de investigación y sexología, se llevó a cabo una validación cuantitativa del contenido de los ítems, en la que se evaluó claridad, relevancia y representatividad de los mismos. Como indicador cuantitativo, se calculó el coeficiente de validez de contenido (CVC). Siguiendo los pasos recomendados por Hernández-Nieto (2002), se calculó el CVC del ítem a partir de las puntuaciones de los expertos que evaluaron con independencia cada ítem según una escala Likert con puntuaciones del 1 al 5 , donde 1 representa la idoneidad más baja del ítem y 5 la más alta. Para hallar el CVC, se dividió la puntuación media obtenida por los expertos en cada ítem por la puntuación máxima que el ítem podría alcanzar, y a este coeficiente se restó el error asignado a cada ítem [error $=(1 / 9)^{9}$, ya que 9 es el número de expertos]. Tal como indica Hernández-Nieto (2002), solo se mantuvieron los ítems cuyo CVC era igual o superior a 0.8. En total, el cuestionario quedó compuesto por 17 ítems que responden a conductas de intimidad física e intimidad emocional. La intimidad física está formada por seis ítems que incluyen el coito, sexo oral, sexo anal y tocamientos. La intimidad emocional contiene 11 ítems y explora conductas relacionadas con los besos, abrazos, caricias, comunicación y masajes. Las respuestas se dan en una escala tipo Likert del 1 al 5, donde 1 indica nunca y 5 siempre. 


\section{Escala triangular del amor de Sternberg}

En la escala original elaborada por Sternberg (1986), el análisis factorial aportó la existencia de tres factores (Compromiso, Pasión e Intimidad). Esta escala fue traducida y aplicada en España por Serrano y Carreño (1993). La validez de constructo que se realizó a través del análisis factorial mostró tres factores que explicaron el $60 \%$ de la varianza total. El alpha de Cronbach osciló en las subescalas entre 0.93 y 0.96 , siendo 0.97 para la escala total. En esta investigación se empleó la Escala Triangular del Amor de Sternberg de tres elementos: Intimidad, Pasión y Compromiso, que consta de 45 ítems, 15 por cada uno de los tres componentes. Se puntúa cada ítem en una escala de nueve puntos, donde 1 era nada y 9 muchísimo. La fiabilidad total de la escala para este estudio fue $\alpha=0.983$. Para la subescala Intimidad, $\alpha=0.952$; para la subescala Pasión, $\alpha$ $=0.942$ y para la subescala Compromiso arrojó un $\alpha=0.95$.

\section{Celos y creencias sobre el amor romántico}

Estos enunciados fueron elaborados por las autoras del artículo; son ítems extraídos de diferentes estudios realizados en España sobre las creencias y mitos del amor romántico (Ferrer et al., 2010; Pascual, 2016; Rodríguez, Lameiras, \& Carrera, 2015). Se halló el Coeficiente de validez de contenido (CVC) de cada ítem, a través de nueve expertos en metodología de investigación y sexología, y se seleccionaron para el estudio los que obtuvieron un CVC igual o superior a 0.8. Se seleccionó un total de ocho ítems. Los ítems sobre celos hacen referencia a la inquietud de la persona amada respecto de que se le reste atención a favor de otra persona: "Soy celoso", "La idea de que mi pareja (AMB y/o Casual) mantenga relaciones con otras personas me haría sentir muy mal", "Me produce ansiedad saber que mi AMB y/o pareja casual muestre más interés por otra persona que por mî". Los ítems que hacen referencia a las creencias sobre amor romántico son: "Creo que hay un solo amor verdadero", "El amor en una relación ayuda a vencer obstáculos", "Creo en el amor a primera vista", "En mi relación de pareja me gusta sentirme especial y único", "Lo importante en una relación de pareja es el sexo". La opción de respuesta es dicotómica.

\section{Procedimiento}

Se presenta un diseño transversal descriptivo en el que la recogida de información se realizó mediante una encuesta en línea, que se difundió enviando el enlace a diferentes listas de asociaciones de jóvenes para que fuese puesto en sus páginas web, y a través de las plataformas virtuales de aprendizaje de profesores universitarios de las áreas de conocimiento de Ciencias Sociales y Jurídicas y Ciencias de la Salud, de 12 universidades españolas. A los participantes se les pidió colaboración para esta investigación y se les instó a compartirla con otros internautas con el objetivo de difundirla. El cuestionario en línea estuvo disponible durante siete meses. Para evitar duplicidades en las respuestas se utilizaron controles de captación, en concreto, el sistema registraba información del ordenador desde el que se accedía al cuestionario y el envío de cookies, que impedía un nuevo acceso. Los participantes fueron informados de ello.

\section{Aspectos éticos}

Los participantes recibieron información sobre el objetivo de la investigación y sobre la institución académica responsable del estudio. Se les comunicó que la participación era voluntaria, la encuesta totalmente anónima y confidencial, y que los datos se utilizarían únicamente por los responsables de la investigación para fines exclusivamente científicos. Se les instó a la sinceridad y honestidad en las respuestas y el derecho a cancelar su participación en cualquier momento. Un requisito imprescindible para acceder al cuestionario era tener como mínimo 18 años de edad. Este estudio fue aprobado por el Comité de Bioética de la Universidad de Almería. 
Análisis de datos

Se realizó un estudio descriptivo de los datos. Una vez comprobado que los datos no se ajustaban a una curva normal, se procedió a realizar contrastes no paramétricos. Para el contraste entre dos grupos independientes, se aplicó el estadístico U de Mann-Whitney. Para comprobar el tamaño del efecto entre los resultados obtenidos, se calculó, por no ajustarse los datos a una curva normal, la $r$ de Rosenthal (1991) y se compararon los resultados, aplicando la clasificación de Cohen (1988) que indica que entre 0.2 y 0.5 , el tamaño del efecto será pequeño, entre 0.5 y 0.8 , será moderado y por encima de 0.8 , será grande. Por último, para conocer los celos y las creencias en el amor en estos dos tipos de relaciones, se llevaron a cabo regresiones logísticas, considerando variable dependiente la respuesta de los participantes a manifestar o no la creencia. Como variables de control e independientes (VI), se tiene el tipo de relación (Relaciones casuales-RC-/Amigos con beneficios-AMB-) y el sexo (hombre/mujer).

\section{Resultados}

\section{Comportamientos sexuales}

La mayoría de los comportamientos emocionales y sexuales aparecen con puntuaciones medias más altas en las relaciones de amigos con beneficios (AMB). Siendo estadísticamente significativas, tanto las conductas que denotan cariño y afecto como las estrictamente sexuales como acariciar los pechos, el coito, tocamientos parcialmente desnudos, tocamientos totalmente desnudos, tocamientos de los genitales, sexo oral y besos en los senos o pecho. Únicamente, salir de inmediato después de tener sexo ha sido significativamente mayor para los socios casuales. El tamaño del efecto calculado en las conductas cuyo resultado fue estadísticamente significativo, fue pequeño, excepto en el comportamiento "hacer un masaje" $(r=0.54)$, en que se considera moderado.
Tabla 1

Comportamientos sexuales y emocionales

\begin{tabular}{|c|c|c|c|c|c|c|c|c|}
\hline \multirow{2}{*}{ Variables } & \multicolumn{2}{|c|}{$M$} & \multicolumn{2}{|c|}{$D S$} & \multicolumn{4}{|l|}{$\mathrm{U}$} \\
\hline & AMB & RSC & AMB & $\mathrm{RSC}$ & $\mathrm{U}$ & $Z$ & $p$ & $r$ \\
\hline Besar en los labios & 4.45 & 4.55 & 0.955 & 0.88 & 2485.5 & -0.495 & 0.621 & \\
\hline Dar la mano & 3.05 & 3.3 & 1.59 & 1.5 & 2341 & -0.967 & 0.333 & \\
\hline Besar en el cuello & 4.3 & 4.13 & 1.03 & 1.2 & 2385 & -0.772 & 0.44 & \\
\hline Dar abrazos y caricias & 4.02 & 3.13 & 1.29 & 1.32 & 1609.5 & -3.796 & $0^{\mathrm{a}}$ & 0.31 \\
\hline Acariciar los pechos & 4.13 & 3.28 & 1.14 & 1.37 & 1613.5 & -3.671 & $0^{\mathrm{a}}$ & 0.29 \\
\hline $\begin{array}{l}\text { Besar alguna parte del } \\
\text { cuerpo }\end{array}$ & 4.53 & 3.96 & 0.85 & 1.17 & 1870 & -3.161 & $0^{\mathrm{a}}$ & 0.25 \\
\hline Penetración /coito & 4.53 & 3.45 & 0.96 & 1.54 & 1491.5 & -4.852 & $0^{\mathrm{a}}$ & 0.38 \\
\hline $\begin{array}{l}\text { Tocamientos } \\
\text { parcialmente desnudos }\end{array}$ & 4.53 & 3.83 & 0.89 & 1.21 & 1645 & -3.92 & $0^{\mathrm{a}}$ & 0.31 \\
\hline $\begin{array}{l}\text { Tocamientos totalmente } \\
\text { desnudos }\end{array}$ & 4.56 & 3.49 & 0.89 & 1.42 & 1455 & -4.946 & $0^{\mathrm{a}}$ & 0.39 \\
\hline Tocar genitales & 4.54 & 3.6 & 0.92 & 1.39 & 1434.5 & -4.887 & $0^{a}$ & 0.39 \\
\hline Sexo oral & 4 & 2.98 & 1.45 & 1.62 & 1629 & -3.885 & $0^{\mathrm{a}}$ & 0.31 \\
\hline Sexo anal & 2.1 & 1.7 & 1.59 & 1.31 & 2169 & -1.577 & 0.115 & \\
\hline $\begin{array}{l}\text { Hablar después de tener } \\
\text { sexo }\end{array}$ & 4.39 & 3.57 & 0.97 & 1.19 & 1477 & -4.502 & $0^{a}$ & 0.36 \\
\hline $\begin{array}{l}\text { Salir inmediatamente } \\
\text { después de tener sexo }\end{array}$ & 1.52 & 1.91 & 0.99 & 1.05 & 1794 & -3.003 & $0.003^{b}$ & 0.24 \\
\hline $\begin{array}{l}\text { Besar en el pecho o } \\
\text { senos }\end{array}$ & 4.15 & 3.21 & 1.22 & 1.33 & 1482 & -4.326 & $0^{a}$ & 0.34 \\
\hline Abrazar & 4.27 & 3.3 & 1.1 & 1.31 & 1478 & -4.512 & $0^{\mathrm{a}}$ & 0.36 \\
\hline Hacer masaje & 3.67 & 1.91 & 1.29 & 1.08 & 831 & -6.856 & $0^{\mathrm{a}}$ & 0.54 \\
\hline
\end{tabular}

Los tipos de amor

Los resultados de la escala de Sternberg indican que la intimidad, la pasión y el compromiso, fueron más intensas en AMB. Las diferencias fueron estadísticamente significativas en pasión e intimidad, aunque el tamaño del efecto, en ambos componentes, fue pequeño, como se observa en la Tabla 2.

Tabla 2

Componentes del amor

\begin{tabular}{|c|c|c|c|c|c|c|c|c|}
\hline \multirow[t]{2}{*}{ Variables } & \multicolumn{2}{|c|}{ Media } & \multicolumn{2}{|c|}{$\begin{array}{l}\text { Desviación } \\
\text { Estándar }\end{array}$} & \multirow[t]{2}{*}{$\mathbf{U}$} & \multirow[t]{2}{*}{$Z$} & \multirow[t]{2}{*}{$p$} & \multirow[t]{2}{*}{$r$} \\
\hline & AMB & RSC & AMB & RSC & & & & \\
\hline Intimidad & 5.62 & 4.66 & 1.74 & 1.99 & 995 & -2.342 & $0.019^{*}$ & 0.21 \\
\hline Pasión & 5.94 & 5.12 & 1.52 & 1.74 & 1104 & -2.175 & $0.03 *$ & 0.2 \\
\hline Compromiso & 5.17 & 4.9 & 1.72 & 1.9 & 1268.5 & -0.62 & 0.535 & \\
\hline
\end{tabular}

Celos y creencias sobre el amor

Se llevaron a cabo ocho modelos de regresión logística, considerando variables dependientes a cada uno de los ocho ítems formulados sobre celos y creencias en el amor romántico, y como predictores, el tipo de relación (RC/AMB) y el sexo (H/M). La prueba de inferencia de Hosmer y Lemeshow no fue significativa en ninguna de las ocho regresiones realizadas, lo que indica que los datos encajan adecuadamente en el modelo. 
Los resultados de las ocho regresiones (Tabla 3) muestran que la variable predictora que mejor evalúa la relación con la variable fidelidad y la variable exclusividad, fue el tipo de relación (RC/AMB), en ambos análisis $p<0$ y $p<$ 0.05 , respectivamente. Los involucrados en $\mathrm{AMB}$ tienen un odds radio de 5.685, en el sentimiento de fidelidad y 2.169 en el de exclusividad, es decir, hay más posibilidad en las relaciones de AMB de sentirse mal por las relaciones de sus parejas con otros y de sentir ansiedad si la pareja muestra interés por otras personas que en aquellos que mantienen relaciones sexuales casuales de una noche.

En lo que se refiere a la creencia romántica de amor a primera vista, la variable predictora que mejor distingue esta creencia también fue el tipo de relación AMB $(p<0.05$ y odds radio de 2.303). En otras palabras, los involucrados en una relación de AMB tienen una posibilidad 2.3 veces mayor de creer en el amor a primera vista que la persona que mantiene relaciones sexuales casuales. En cambio, la variable predictora que mejor distingue la creencia de sentirse especiales y únicos en la relación de pareja, es la variable sexo ( $p<0.05$ y odds radio 0.309 ), al ser odds radio menor que 1 , la interpretación debemos hacerla en sentido inverso, es decir, 1/0.309 es igual a 3,23, es decir, hay 3.23 veces menos posibilidad que una mujer muestre la creencia (ya esté involucrada en $\mathrm{AMB}$ o $\mathrm{RC}$ ) que un hombre.
Tabla 3

Regresiones logísticas de las creencias sobre el amor romántico

\begin{tabular}{|c|c|c|c|c|}
\hline Creencias & VI o predictora & $\beta$ & Odds radio & I.C. $95 \%$ \\
\hline \multirow{5}{*}{$\begin{array}{l}\text { Me considero una persona } \\
\text { celosa }\end{array}$} & Tipo de relación RC/AMB & 0.181 & 1.199 & $0.653-2.443$ \\
\hline & Sexo $H / M$ & 0.233 & 1.263 & \multirow{4}{*}{$0.599-2.4$} \\
\hline & Constante & -0.433 & \multirow[t]{3}{*}{0.649} & \\
\hline & $2 \mathrm{log}$. verosimilitud & 228.13 & & \\
\hline & $\mathrm{R}^{2}$ de Nagelkerke & 0.006 & & \\
\hline La idea de que mi pareja & Tipo de relación RC/AMB & 1.738 & $5.685^{* * *}$ & 2.243-14.408 \\
\hline mantenga relaciones con & Sexo $H / M$ & -0.53 & 0.588 & \multirow[t]{4}{*}{$0.277-1.25$} \\
\hline otros me haria sentirme & Constante & 0.472 & \multirow[t]{3}{*}{1.603} & \\
\hline \multirow[t]{2}{*}{ muy mal } & 2 log. verosimilitud & 194.64 & & \\
\hline & $\mathrm{R}^{2}$ de Nagelkerke & 0.151 & & \\
\hline Me produce ansiedad el & Tipo de relación RC/AMB & 0.774 & 2.169* & $1.073-4.385$ \\
\hline hecho de saber que mi & Sexo $H / M$ & -0.518 & 0.596 & $0.295-1.203$ \\
\hline pareja mostrara más & Constante & 0.292 & 1.34 & \\
\hline interés por otra pareja que & $2 \mathrm{log}$, verosimilitud & 222.573 & & \\
\hline por $\mathrm{mi}$ & $R^{2}$ de Nagelkerke & 0.055 & & \\
\hline \multirow{5}{*}{$\begin{array}{l}\text { Creo que hay un solo amor } \\
\text { verdadero }\end{array}$} & Tipo de relación RC/AMB & -0.274 & 0.76 & $0.347-1.664$ \\
\hline & Sexo $H / M$ & 0.059 & 1.061 & \multirow{4}{*}{$0.478-2.354$} \\
\hline & Constante & -1.08 & 0.339 & \\
\hline & 2 log. verosimilitud & 187.321 & & \\
\hline & $R^{2}$ de Nagelkerke & 0.004 & & \\
\hline \multirow{5}{*}{$\begin{array}{l}\text { El amor en una relación } \\
\text { ayuda a vencer obstáculos }\end{array}$} & Tipo de relación RC/AMB & 0.325 & 1.384 & \multirow{5}{*}{$\begin{array}{r}0.43 /-4.033 \\
0.364-3.14\end{array}$} \\
\hline & Sexo $H / M$ & 0.067 & 1.069 & \\
\hline & Constante & 1.9 & 6.683 & \\
\hline & 2 log. verosimilitud & 122.519 & & \\
\hline & $R^{2}$ de Nagelkerke & 0.004 & & \\
\hline \multirow{5}{*}{$\begin{array}{l}\text { Creoo en el amor a primera } \\
\text { vista }\end{array}$} & Tipo de relación RC/AMB & 0.834 & 2.303* & $1.165-4.554$ \\
\hline & Sexo $H / M$ & -0.085 & 0.918 & \multirow[t]{4}{*}{$0.456-1.849$} \\
\hline & Constante & -0.063 & 0.939 & \\
\hline & 2 log, verosimilitud & 228.434 & & \\
\hline & $\mathrm{R}^{2}$ de Nagelkerke & 0.047 & & \\
\hline En mi relación me gusta & Tipo de relación RC/AMB & -0.258 & 0.773 & \multirow{5}{*}{$0.101-0.939$} \\
\hline sentirme especial y & Sexo $H / M$ & -1.176 & $0.309^{*}$ & \\
\hline \multirow[t]{3}{*}{ única/o } & Constante & 2.922 & 18.57 & \\
\hline & 2 log. verosimilitud & 92.442 & & \\
\hline & $\mathrm{R}^{2}$ de Nagelkerke & 0.057 & & \\
\hline \multirow{5}{*}{$\begin{array}{l}\text { relación es el sexo } \\
\text { relate }\end{array}$} & Tipo de relación RC/AMB & 0.388 & & \multirow{5}{*}{$\begin{array}{r}0.119-3.023 \\
0.59-2.737\end{array}$} \\
\hline & Sexo $H / M$ & 0.239 & 1.27 & \\
\hline & Constante & -1.199 & 0.302 & \\
\hline & 2 log. verosimilitud & 193.803 & & \\
\hline & $\mathrm{R}^{2}$ de Nagelkerke & 0.013 & & \\
\hline
\end{tabular}

\section{Debate y conclusiones}

Este estudio confirma que se está asistiendo a cambios en la forma de entender el amor y las relaciones de pareja. Analiza las relaciones de amigos con beneficios y el sexo ocasional, y profundiza en los comportamientos sexuales, el tipo de amor y las creencias sobre el amor romántico que manifiestan los involucrados en ambas relaciones. En esta investigación, el número de participantes involucrados en una relación de $\mathrm{AMB}$ es superior a los involucrados en el sexo casual con extraños, lo que apoya que el sexo sin compromiso se produce con mayor frecuencia con amigos que con extraños (Grello et al., 2006).

El primero de los hallazgos señala que los comportamientos sexuales físicos son más intensos en las relaciones de $\mathrm{AMB}$ en donde la amistad en curso y la confianza favorece la intimidad física (Owen et al., 2017). Por otra parte, también las conductas sexuales que denotan afectividad y emociones son más intensas en los amigos con beneficios, excepto tres comportamientos afectivos como besar en 
los labios, besar en el cuello y dar la mano que se expresan igual en los dos tipos de relaciones. Debido a la temporalidad de los encuentros, en $\mathrm{AMB}$ se propician más oportunidades para una mayor variedad de prácticas sexuales (Jonason et al., 2011). Estos actos fluctúan desde caricias, besos y tocamientos, hasta sexo oral y/ o vaginal (Bisson \& Levine, 2009; Mongeau, Knight, Williams, Eden, \& Shaw, 2013; Owen \& Fincham, 2011a). También son muy comunes las actividades que denotan cercanía emocional y afectiva (besos, caricias, abrazos, cogerse de las manos, masajes, comunicación y pasar tiempo juntos), revelando el carácter más romántico de AMB. En cambio, en el encuentro de una noche, las personas reducen estas conductas priorizando sobre todo el aspecto erótico (Furman \& Shaffer, 2011; Grello et al., 2006; Jonason et al., 2009; Jonason et al., 2011), aunque en este estudio se ha encontrado que conductas afectivas como besos en los labios o en el cuello las realizan tanto los $\mathrm{AMB}$ como los que mantienen relaciones casuales.

En el plano estrictamente sexual, el coito también ha sido significativamente más frecuente cuando el socio ha sido un amigo, y es que los encuentros con más periodicidad generan más oportunidades para que esta conducta se produzca (Furman \& Shaffer, 2011; Jonason et al., 2011). No obstante, no es la actividad sexual más frecuente, ni el encuentro sexual implica necesariamente el coito (Olmstead, Pasley, \& Fincham 2013), contrariamente a las tesis mantenidas por otros autores (Bisson \& Levine, 2009; Furman \& Shaffer, 2011; Mongeau et al., 2013; Owen \& Fincham, 2011a). Los resultados muestran que aun dándose las conductas sexuales físicas y emocionales en los dos tipos de relaciones, lo hace con mayor frecuencia en $\mathrm{AMB}$, lo que apoya parcialmente la primera hipótesis formulada.

Las respuestas de los participantes en esta investigación dan a conocer los comportamientos sexuales en las relaciones de amigos con beneficios y en la práctica de sexo casual, pero se coincide con Margansk y Fauth (2013), en la dificultad para precisar qué conductas sexuales y emocionales son típicas de cada una de las relaciones estudiadas.

En relación con el amor, los tres componentes son más profundos en AMB, destacando sobre todo la intimidad y la pasión, dos elementos del amor donde los resultados son estadísticamente significativos, coincidiendo con estudios realizados en la última década (Lehmiller et al., 2011; McGinty et al., 2007; Owen \& Fincham, 2011a). El sexo centrado en el placer mejora la satisfacción cuando se asocia con la intimidad (Rodrigue \& Fernet, 2016). Esto también confirma que las personas persiguen algo más que una meta exclusivamente sexual (Bisson \& Levine, 2009). Además, el resultado es coherente con los que indican que puesto que AMB combina características de amistad, relación romántica y sexo casual, la pasión y la intimidad son más notorias que en el sexo ocasional (Birnie-Porter \& Hunt, 2015; Bisson \& Levine, 2009; Lehmiller et al., 2011; McGinty et al., 2007; Owen \& Fincham, 2011a). Siguiendo la teoría de Sternberg (1986), el tipo de amor característico de AMB muestra altas dosis de atracción física y deseo erótico, un sentimiento de cercanía y confianza, la pareja está unida pasional y afectivamente, pero no hay compromiso de exclusividad y fidelidad. Estos hallazgos difieren de los de Bisson y Levine (2009), quienes encontraron que en $\mathrm{AMB}$ la intimidad es elevada, pero baja la pasión y el compromiso, por lo que sería un estilo de amor únicamente amistoso. Por otro lado, la relación sexual casual con extraños o poco conocidos se comporta acorde a su naturaleza, caracterizándose por la activación sexual y bajos niveles de confianza, intimidad, cariño y compromiso (Hatfield et al., 2012; Smith et al., 2012). Los resultados apoyan la segunda hipótesis.

Como ya se ha comentado al comienzo del artículo, se vive en un mundo individualista, consumista e inmerso en cambios vertiginosos, donde aparecen nuevos tipos de relaciones distantes de las tradicionales, incluso en el amor y relación de pareja (Bauman, 2001; Blandón-Hincapié \& López-Serna, 2016). En esta investigación, se comprueba qué queda de las creencias del amor romántico, y en qué 
tipo de relación se aprecia con más fuerza. La fidelidad y exclusividad en las relaciones son más intensas en AMB que en el sexo ocasional. Los individuos muestran mayor pesar al pensar en la transgresión de la fidelidad, lo que corrobora que en $\mathrm{AMB}$ los sujetos deben mantener algún tipo de compromiso que les hace expresar esta emoción (Bisson \& Levine, 2009). Aparecen, en esta relación, elementos de lealtad y exclusividad propios del vínculo romántico (Yela, 2003). En cuanto a la creencia del amor a primera vista es más intensa su posibilidad en $\mathrm{AMB}$ que en la relación meramente casual. Sin embargo, el inicio de AMB no está motivado exclusivamente por el deseo sexual, sino que median otros elementos como pueden ser los beneficios asociados a la amistad, el soporte afectivo y el social (Gusarova et al., 2012). En cambio, en el tipo de relaciones casuales de una noche y en AMB, la creencia romántica de sentirse especiales y únicos en la relación ya no se atribuye a las mujeres, como tradicionalmente se ha hecho (Ferrer et al., 2010; Pascual, 2016), sino que en este caso esta creencia es más intensa en el hombre. Este resultado no apoya por completo la última hipótesis, que siguiendo la tradición se piensa que las mujeres son las que pretenden mantener la atención del hombre (Ferrer \& Bosch, 2013). Los resultados muestran que con los cambios sociales se está produciendo un acercamiento entre sexos (Reid, Elliott, \& Webber, 2011).

Hemos visto que también que las creencias que tradicionalmente han estado asociadas a las relaciones amorosas estables, aparecen en el nuevo tipo de relación de $\mathrm{AMB}$, lo que refuerza el carácter híbrido de este vínculo (Bisson \& Levine, 2009), y permite afirmar que AMB presenta características de la relación romántica, distanciándose de la perspectiva pragmática de las relaciones casuales, contrariamente a lo expuesto por algunos autores (Puentes, Knox, \& Zusman, 2008).

Coincidiendo con Illouz (2009), se señala que no se puede postular un cambio radical en las vivencias amorosas, y es necesario matizar la idea de la desaparición del amor romántico, ya que actualmente conviven visiones prácticas e igualitarias del amor con otras pasionales con aspiraciones románticas. En resumen, los resultados de este estudio refuerzan el carácter híbrido de amigos con beneficios, a medio camino entre el vínculo puramente sexual y la relación romántica.

En cuanto a las limitaciones del presente estudio, se destacan las dificultades para acceder a la muestra y encontrar personas que reconozcan estar involucradas en AMB o en una relación sexual de una noche; probablemente, la propia fugacidad de este tipo de relaciones lo dificulte o bien la evitación a tratar estos temas. Por otra parte, hay una falta de homogenización de las muestras en cuanto al número de personas implicadas en cada relación, todo ello exige cautela en la interpretación de los datos y la generalización de los resultados.

Como líneas para futuras investigaciones, se señala que es necesario profundizar en este campo con el objeto de facilitar la comprensión de estos vínculos sexuales y afectivos, e ir generando conocimiento sobre las relaciones sexuales del siglo XXI. Quedan muchos aspectos por descubrir, son indispensables trabajos que ofrezcan clarificación teórica y ayuden a interpretar estos novedosos universos sexuales y afectivos. Es necesario examinar las definiciones, con el fin de conseguir una precisión y unanimidad terminológica para referirse a estas relaciones casuales. AMB plantea diferentes incógnitas por descubrir en futuros estudios: ¿Por qué los individuos se involucran en estas relaciones? ¿Qué beneficios obtienen? ¿Qué grado de amistad hay que tener para considerarse un AMB? ¿Cómo se comportan hombres y mujeres? ¿Cómo incide este tipo de relaciones en la satisfacción sexual? ¿Cómo afecta la aceptación social a la persona involucrada en este tipo de relación? ¿Pueden psicológicamente desencadenar algún conflicto en la persona? ¿Cómo afecta la relación a la autoestima? ¿Qué papel desempeña la religiosidad en estas relaciones? En definitiva, continuar indagando en lo que Santos Vega (2009) denominó "follamistad", haciendo referencia a un marco relacional que empieza a operar en la vida cotidiana, y que cuenta cada vez con un mayor 
número de practicantes, pero que va más allá del acto sexual.

\section{Referencias}

Akbulut, V., \& Weger, H. (2016). Predicting responses to bids for sexual and romantic escalation in cross-sex friendships. The Journal of Social Psychology, 156, 98-114. https://doi.org/10.1080/00224545. 2015.1066296

Bauman, Z. (2001). La sociedad individualizada. Madrid: Cátedra.

Bauman, Z. (2005). Amor líquido. Acerca de la fragilidad de los vínculos humanos. México: Fondo de Cultura Económica.

Birnie-Porter, C., \& Hunt, M. (2015). Does relationship status matter for sexual satisfaction? The roles of intimacy and attachment avoidance in sexual satisfaction across five types of ongoing sexual relationships. The Canadian Journal of Human Sexuality, 24(2), 174-183. https://d oi.org/10.3138/cjhs.242-A5

Bisson, M. A., \& Levine, T. R. (2009). Negotiating a friends with benefits relationship. Archives of Sexual Behavior, 38(1), 66-73. https://doi.org/10.1007/s1050 8-007-9211-2

Blandón-Hincapié, A. I., \& López-Serna, L. M. (2016).Comprensiones sobre pareja en la actualidad: jóvenes en busca de estabilidad. Revista Latinoamericana de Ciencias Sociales, Ninez y Juventud, 14(1), 505-517. https://d oi.org/10.11600/1692715x.14134271014

Claxton, S. E., \& van Dulmen, M. H. (2013).Casual sexual relationships and experiences in emerging adulthood. Emerging Adulthood, 1(2), 138-150. https:// doi.org/10.1177/2167696813487181

Cohen, J. (1988). Statistical power analysis for the behavioral sciences. Hilsdale, NJ: Earlbaum.

de Olalla, P. G. (2007). Comentario. «Amigos con derecho a roce». Gaceta Sanitaria, $21(6)$, 479. https://doi.org/10.1157/13112241

Dube, S., Lavoie, F., Blais, M., \& Hebert, M. (2017). Psychological well-being as a predictor of casual sex relationships and experiences among adolescents: A shortterm prospective study. Archives of Sexual Behavior, 46, 1807-1818. https://doi.org/10. 1007/s10508-016-0914-0

Eisenberg, M. E., Ackard, D. M., Resnick, M. D., \& Neumark Sztainer, D. (2009). Casual sex and psychological health among young adults: Is having "friends with benefits" emotionally damaging? Perspectives on Sexual and Reproductive Health, 41(4), 231-237. https://doi.org/10.1363/4123109

Fernández-Dávila, P. (2007). «Amigos con derecho a roce»: una oportunidad para contraer la infección por el virus de la inmunodeficiencia humana en hombres homo/bixesuales con prácticas sexuales de alto riesgo. Gaceta Sanitaria, 21(6), 471-478. https://doi.org/10.1157/13112240

Ferrer, V. A., Bosch, E., \& Navarro, C. (2010). Los mitos románticos en España. Boletín de Psicología, 99, 7-31. https://www.uv.es/seoa ne/boletin/previos/N99-1.pdf

Ferrer, V., \& Bosch, E. (2013). Del amor romántico a la violencia de género. Para una coeducación emocional en la agenda educativa. Profesorado. Revista de Currículum y Formación de Profesorado, 17(1), 105-122. Recuperado de https://ww w.ugr.es/ recfpro/rev171ART7.pdf

Fuente, M. (2010). El resurgir de la sífilis. Actas Dermosifiliograficas, 101 (10), 817-819. http s://doi.org/10.1016/j.ad.2010.10.001

Furman, W., \& Shaffer, L. (2011). Romantic partners, friends, friends with benefits, and casual acquaintances as sexual partners. Journal of Sex Research, 48(6), 554-564. http s://doi.org/10.1080/00224499.2010.535623

García, J. R., \& Reiber, C. (2008). Hook-up behavior: A biopsychosocial perspective. Journal of Social, Evolutionary, and Cultural Psychology, 2(4), 192-208. https://doi.org/1 $0.1037 / h 0099345$

García-Serrán, H., \& Soriano-Ayala, E. (2016). "Amigos con beneficios": salud sexual y estilos de apego de hombres y mujeres. Saúde e Sociedade, 25 (4), 1136-1147. https: //doi.org/10.1590/s0104-12902016151111 
Gomes, A. C., Caramelo, F., Patricio, M., Camarneiro, A. P., Massano, S., \& Rui, J. (2017). Impacto de un programa de intervención educativa en los comportamientos sexuales de jóvenes universitarios. Enfermagem, 13, 71-82. http s://doi.org/10.12707/RIV17022

Grello, C. M., Welsh, D. P., \& Harper, M. S. (2006). No strings attached: The nature of casual sex in college students. Journal of Sex Research, 43, 255-267. https://doi.org/10.10 $80 / 00224490609552324$

Gusarova, I., Fraser, V., \& Alderson, K. G. (2012). A quantitative study of 'friends with benefits' relationships. Canadian Journal of Human Sexuality, 21(1), 41-59. Recuperado de http://www.researchgate.net/publicatio n/288701935_A_quantitative_study_of_fr iends_with_benefits_relationships

Hatfield, E., Hutchison, E. S. S, Bensman, L., Young, D., \& Rapson, R. L. (2012). Cultural, social, and gender influences on casual sex: New developments. En J. M. Turner \& A. D. Mitchell (Eds.), Social psychology: New developments (pp. 1-37). Hauppauge, NY: Nova Science.

Hernández González, J., \& Weiss, E. (2010, octubre). Amor y sexualidad entre jóvenes de la escuela media en México. Cuadernos de Educación, año VIII, núm. 8, pp. 245-256. http://revistas.unc.edu.ar/index.p $\mathrm{hp} /$ Cuadernos/article/view/810/763

Hernández-Nieto, R. A. (2002). Contribuciones al análisis estadístico de datos. Mérida, VE: Universidad de Los Andes.

Illouz, E. (2009). El consumo de la utopía romántica. Madrid/Buenos Aires: Katz Editores.

Jonason, P. K., Li, N. P., \& Cason, M. J. (2009). The "booty call": A compromise between men's and women's ideal mating strategies. Journal of Sex Research, 46(5), 460-470. htt ps://doi.org/10.1080/00224490902775827

Jonason, P. K., Li, N. P., \& Richardson, J. (2011). Positioning the booty-call relationship on the spectrum of relationships: Sexual but more emotional than one-night stands.
Journal of Sex Research, 48(5), 486-495. http s://doi.org/10.1080/00224499.2010.497984

Lehmiller, J. J., VanderDrift, L. E., \& Kelly, J. R. (2011). Sex differences in approaching friends with benefits relationships. The Journal of Sex Research, 48(2-3), 275-284. ht tps://doi.org/10.1080/00224491003721694

Lehmiller, J. J., VanderDrift, L. E., \& Kelly, J. R. (2014). Sexual communication, satisfaction, and condom use behavior in friends with benefits and romantic partners. Journal of Sex Research, 51(1), 74-85. https: //doi.org/10.1080/00224499.2012.719167

Letcher, A., \& Carmona, J. (2015). Friends with Benefits: Dating practices of rural high school and college students. Journal Community Health, 40, 522-529. https://doi .org/10.1007/s10900-014-9966-z

Luiz de Andrade, A., \& Wachelke, J. (2011). La asociación de configuraciones estructurales de las relaciones románticas con las creencias sobre las relaciones de pareja: un estudio de las representaciones sociales. Anales de Psicología, 27(3), 834-842. Recuperado de https://www.academia.edu/899863/The _association_of_structural_configurations _of_romantic_relationships_with_beliefs_ about_couple_relationships_a_social_repr esentations study

Lyons, H., Manning, W., Giordano, P., \& Longmore, M. (2010). Casual sex among young adults: Education differentials. Papeles de trabajo del Center for Family and Demographic Research (Working Paper Series 2010-07). Bowling Green, OH: Bowling Green State University. Recuperado de https://pdfs.semanticscholar.org/a39d/a 98cca64e1ec5f6b99b1aa17df39b75a4bf9.p df

Macchi, M., Benítez Leite, S., Núñez C., \& Ortigoza, D. (2008). Conocimientos, actitudes y prácticas acerca del VIH/ SIDA en jóvenes de nivel medio de educación, del área metropolitana, Paraguay. Revista Chilena De Pediatría, 
79(2), 206-217. https://doi.org/10.4067/SO 370-41062008000200012

Manning, W. D., Giordano, P. C., \& Longmore, M. A. (2006). Hooking up the relationship contexts of "nonrelationship" sex. Journal of Adolescent Research, 21(5), 459-483. https:/ /doi.org/10.1177/0743558406291692

Marganski, A., \& Fauth, K. (2013). Hooking up in the Twenty-First Century. Social Crimonol 1(1), 2-7. https://doi.org/10.4172/2375-443 5.1000102

Marquard, O. (2001). Filosofía de la compensación. Barcelona: Paidós.

McGinty, K., Knox, D., \& Zusman, M. E. (2007). Friends with benefits: Women want "friends," men want "benefits". College Student Journal, 41, 1128-1131. (N. ${ }^{\circ}$ de servicio de reproducción de documentos ERIC EJ 816 839)

Mongeau, P. A., Knight, K., Williams, J., Eden, J., \& Shaw, C. (2013). Identifying and explicating variation among friends with benefits relationships. Journal of Sex Research, 50(1), 37-47. https://doi.org/10.1 080/00224499.2011.623797

Olmstead, S. B., Pasley, K., \& Fincham, F. D. (2013). Hooking up and penetrative hookups: Correlates that differentiate college men. Archives of Sexual Behavior, 42 (4), 573-583. https://doi.org/10.1007/s10 508-012-9907-9

Owen, J., \& Fincham, F. D. (2011a). Effects of gender and psychosocial factors on "friends with benefits" relationships among young adults. Archives of Sexual Behavior, 40(2), 311-320. https://doi.org/10.1007/s10508-0 10-9611-6

Owen, J., \& Fincham, F. D. (2011b). Young adults' emotional reactions after hooking up encounters. Archives of Sexual Behavior, 40 (2), 321-330. https://doi.org/10.1007/s10 508-010-9652-x

Owen, J., Fincham, F. \& Polser, G. (2017). Couple identity, sacrifice, and availability of alternative partners: Dedication in friends with benefits relationships. Archives of Sexual Behavior, 46, 1785-1791. https://doi. org/10.1007/s10508-016-0716-4
Pascual, A. (2016). Sobre el mito del amor. Amores cinematográficos y educación. DEDICA, Revista de Educaçao e Humanidades, 10, 63-78. Recuperado de https://dialnet.unirioja.es/servlet/articul o? codigo $=5429358$

Puentes, J., Knox, D., \& Zusman, M. E. (2008). Participants in "friends with benefits" relationships. College Student Journal, 42, 176-180. (N..$^{\circ}$ de servicio de reproducción de documentos ERIC EJ 816 877)

Quiñones, R., Martínez-Taboas, J. R., RodríguezGómez, J. R., \& Pando, C. (2017). Friends with benefits in Puerto Rican college students. Revista Interamericana de Psicología, 51(1), 19- 28. Recuperado de https://psycnet.apa.org/record/2018-239 50-002

Quirk, K., Owen, J., \& Fincham, F. (2014). Perceptions of partner's deception in friends with benefits relationships, Journal of Sex $\mathbb{E}$ Marital Therapy, 40(1), 43-57. https://doi.o $\mathrm{rg} / 10.1080 / 0092623 X .2012 .668513$

Reid, J. A., Elliott, S., \& Webber, G. R. (2011). Casual hook-ups to formal dates refining the boundaries of the sexual double standard. Gender $\mathcal{E}$ Society, 25(5), 545-568. https://doi.org/10.1177/08912432 11418642

Rodrigue, C., \& Fernet, M. (2016). A metasynthesis of qualitative studies on casual sexual relationships and experiences. The Canadian Journal of Human Sexuality, 25(3), 225-242. https://doi.org/10.3138/cjh s.253-A6

Rodríguez, Y., Lameiras, M., \& Carrera, M. V. (2015). Amor y sexismo: una peligrosa relación en los y las adolescentes gallegos/ as. Revista de Estudios e Investigación en Psicología y Educación, 2, A2-12. https://doi .org/10.17979/reipe.2015.0.02.234

Rodríguez, A., Hernán, M., García, J. M., \& Romo, N. (2007). ¿Qué opinan adolescentes y jóvenes sobre el consumo de drogas recreativas y las conductas sexuales de riesgo? Adicciones, 19, 153-167. https://d oi.org/10.20882/adicciones.313 
Rosenthal, R. (1991). Meta-analytic procedures for social research (ed. rev.). Newbury Park, CA: Sage. https://doi.org/10.4135/97814129849 97

Santos Vega, J. D. (2009). "Debarías buscar un follamigo": a propósito de la flexibilidad en las relaciones personales. Témpora: Revista de Historia y Sociología de la Educación, 12, 37-64. Recuperado de https://www.academia.edu/10961420/ Deber\%C3\%ADas buscar un follamigo . _A_prop\%C3\%B3sito_de_la_flexibilidad_en_las_relaciones_personales_You_Should find_a_sex_friend_._On_flexibility_in_p ersonal_relationships

Serrano, G., \& Carreño, M. (1993). La teoría de Sternberg sobre el amor. Análisis empírico [Edición especial]. Psicothema, 5, 151-157. Recuperado de https://dialnet.unirioja.es/se rvlet/articulo? codigo $=2018600$

Scott, C. L., Carrillo, B., \& Rivera, I. M. (2014). Exploring undergraduates` sexual decision making in friends with benefits relationships. En J. H. McCormick \& S. L. Blair (Eds.), Family relationships and familial responses to health issues (pp. 31-74). Bradford, UK: Emerald Group Publishing.

Scott, C. L., Hash, J. M., Stevens, P., \& Tejada, T. (2016). Divorcing commitment: Examining the role of parental divorce in undergraduates' friends with benefits relationships. En G. Gianesini \& S. Lee Blair (Eds.), Divorce, Separation, and Remarriage: The transformation of family (pp. 229-250). Emerald Group Publishing. https://doi.org/10.1108/S1530. 353520160000010009

Smith, A. M. A., Patrick, K., Heywood, W., Pitts, M. K., Richters, J., Shelley, J. M.,... Ryall, R. (2012). Body mass index, sexual difficulties and sexual satisfaction among people in regular heterosexual relationships: A population-based study. Internal Medicine Journal, 42 (6), 641-651. https://doi.org/10. 1111/j.1445-5994.2012.02808.x

Sterba, S. K., \& Foster, E. M. (2008). Selfselected sample. En P. J. Lavrakas (Ed.),
Encyclopedia of survey research methods (pp. 806-808). Thousand Oaks, CA: Sage.

Sternberg, R. J. (1986). A triangular theory of love. Psychological Review, 93(2), 119-135. h ttps://doi.org/10.1037/0033-295X.93.2.119

Sternberg, R. J. (1997). Construct validation of a triangular love scale. European Journal of Social Psychology, 27(3), 313-335. https://doi.org/10.1002/(SICI) 10 99-0992(199705)27:33.3.CO;2-W

Suárez, M. (2016). ¿Por qué lo llaman amor cuando quieren decir violencia? Trabajo presentado en el XII Congreso Español de Sociología, Gijón, Asturias. Recuperado de http://www.fes-sociologia.com/files/cong ress/12/papers/3081.pdf

Uribe, J. I., Amador, G., Zacarías, X., \& Villarreal, L. (2012). Percepciones sobre el uso del condón y la sexualidad entre jóvenes. Revista Latinoamericana de Ciencias Sociales, Niñez y Juventud, 10(1), 481-494. Recuperado de http://www.scielo.org.co/pd f/rlcs/v10n1/v10n1a31.pdf

Vizzuetth, A., García, M., \& Guzmán, R. (2010). Expectativas sobre la relación de amigovios, freefly novios en jóvenes adultos. En A. S. Rivera, R. Díaz-Loving, A. R. Sánchez, I. Reyes-Lagunes \& L. Cruz Martínez (Eds.), La psicología social en México (pp. 223-230). Mexico: AMEPSO.

Wentland, J. J., \& Reissing, E. D. (2011). Taking casual sex not too casually: Exploring definitions of casual sexual relationships. Canadian Journal of Human Sexuality, 20(3), 75-91. Recuperado de https://psycnet.apa.o rg/record/2011-30584-003

Wentland, J. J., \& Reissing, E. (2014). Casual sexual relationships: Identifying definitions for one night stands, booty calls, fuck buddies, and friends with benefits. The Canadian Journal of Human Sexuality, 23(3), 167-177. https://doi.org/10.3138/cjhs.2744

Yela, C. (2003). La otra cara del amor: mitos, paradojas y problemas. Encuentros en Psicología Social, 1(2), 263-267.

\section{Notas}

* Artículo de investigación. 